\title{
TRATA DE MUJERES CON FINES DE EXPLOTACIÓN SEXUAL Y VIOLENCIA DE GÉNERO. INDICIOS PARA LA DETECCIÓN DE POSIBLES VÍCTIMAS.
}

\section{TRAFFICKING IN WOMEN FOR SEXUAL EXPLOITATION PURPOSES AND GENDER VIOLENCE. CLUES FOR THE DETECTION OF POSSIBLE VICTIMS.}

\author{
María del Águila Lara Palacios. Universidad Pablo de Olavide de Sevilla - España \\ mlarapalacios@gmail.com \\ Mari Carmen Monreal Gimeno. Universidad Pablo de Olavide de Sevilla - España \\ momongim@upo.es \\ Sebastián Sánchez Fernández. Universidad de Granada - España \\ ssanchez@ugr.es
}

\begin{abstract}
Resumen: La finalidad de la investigación es visibilizar los indicios para detectar e identificar a las mujeres próximas a redes de trata de personas con fines de explotación sexual. Estos indicios son el resultado de la experiencia profesional directa de la investigadora en el CETI de Melilla con mujeres próximas a estas redes, y de sucesivas formaciones que ha realizado. En la introducción se sitúa a las personas lectoras ante el tema de la trata: Cuál es su fin y cuándo empezó a formar parte de la agenda política. En el desarrollo, se exponen los objetivos, los elementos diferenciadores de la definición de trata de personas, así como, las diferencias de este término con el de tráfico ilícito de personas; quién es el organismo competente para la identificación de las víctimas; cómo es la gestión del proceso para su detección; cuál es la legislación nacional necesaria a aplicar ante los casos de trata; $y$, se explican cada uno de los indicios y su importancia para estructurar las entrevistas para la detección de casos de trata. En las conclusiones se exponen de manera concisa cada uno de estos apartados.
\end{abstract}

Palabras clave: Trata de personas con fines de explotación sexual, indicios, violencia de género, tráfico ilícito de personas, entrevistas, detección.

\begin{abstract}
The purpose of the research is to make evidence visible to detect and identify women close to trafficking of human beings networks for sexual exploitation purposes. These indications are the result of the researcher's direct professional experience at Melilla's CETI with women close to these networks, and successive trainings she has made. The introduction places readers on the subject of trafficking: What is its purpose and when it became part of the political agenda. Development sets out the objectives, the differentiating elements of the definition of trafficking in persons, as well as the differences in this term with that of illicit trafficking in persons; who is the body responsible for identifying victims; how process management is for detection; what national legislation is necessary to apply in the face of trafficking of human beings cases; and, each of the indications and their importance in structuring interviews for the detection of trafficking cases are explained. The conclusions set out each of these paragraphs concisely.
\end{abstract}

Keys Word: Trafficking of human beings for sexual exploitation purposes, gender violence, illicit human trafficking, interviews, detection. 


\section{Introducción}

La trata de personas tiene por objetivo la explotación de los seres humanos a cambio del beneficio económico de las personas responsables de esta forma de esclavitud.

Asimismo, el informe del Defensor del Pueblo asegura que las violaciones de derechos humanos por razón de género son una de las causas fundamentales de la existencia de la trata de personas (Defensor del Pueblo, 2015, p.3).

Por otro lado, los Estados Parte sí han considerado las recomendaciones de la Comisión Europea y han elaborado su Plan de Acción contra la trata, como es el caso de España (2007) (2009-2011) (2015-2018).

La lucha contra la trata de seres humanos con fines de explotación sexual empezó a formar parte de la agenda política de las Naciones Unidas en el año 2000, que dio lugar al documento de lucha contra la trata denominado Protocolo para prevenir, reprimir y sancionar la trata de personas, especialmente mujeres y niños, que complementa la Convención de las Naciones Unidas contra la Delincuencia Organizada Transnacional, conocido como "Protocolo de Palermo"."

Por otro lado, algunos Estados Parte han creado un documento para luchar contra la trata de seres humanos siguiendo la recomendación del Protocolo de Palermo (2000) y otros, por el contrario, no han regulado nada al respecto.

Este trabajo surge de la experiencia profesional y directa con la población inmigrante, en la ciudad autónoma de Melilla, de la investigadora, como profesora de Español a personas extranjeras en el $\mathrm{CETI}^{2}$, y como responsable provincial de la asociación Accem en la ciudad.

Por tanto ha podido conocer a mujeres próximas a redes de trata de personas. $\mathrm{Ha}$ tenido la oportunidad de enseñarles Español y su cultura, y en muchas ocasiones también a leer y a escribir. Mediante este contacto directo ha tenido la oportunidad de detectar ciertos indicios que pueden ayudar a las personas competentes a identificar a las personas como víctimas de trata. En la elaboración de este trabajo también se ha tenido en cuenta todas las formaciones que ha recibido por Accem en este tema a tratar.

Estos indicios que no son contemplados, al menos no lo suficiente, en las formaciones impartidas a los/as profesionales en ello, al Cuerpo Nacional de Policía.

Es muy importante conocer todos los indicios posibles para programar las entrevistas a las personas inmigrantes para detectar si se encuentran próximas a redes de trata. Igualmente, estos indicios pueden ser utilizados para cualquier fin de

\footnotetext{
${ }^{1}$ En adelante, Protocolo de Palermo.

${ }^{2}$ CETI: Centro de estancia temporal a inmigrantes.
} 
explotación de la trata de personas, no sólo concierne a la trata con explotación sexual.

Previamente a exponer los indicios detectados, este trabajo pretende situar a las personas lectoras ante la problemática de la trata de personas analizando los elementos identificadores del término trata de personas; la diferencia entre el concepto de trata de personas con el de tráfico ilícito de personas porque en numerosas formaciones que he impartido ambos términos suelen confundirse; así como, cuándo se asume en la agenda política europea y nacional este tema; cómo se gestiona la identificación de las personas víctimas de trata en el Estado Español; y la legislación nacional existente al respecto.

Posteriormente a los indicios, este trabajo termina con las conclusiones obtenidas del mismo.

\section{Desarrollo}

Con este trabajo perseguimos el siguiente objetivo general:

- Realizar una aproximación al tema de la trata de personas con fines de explotación sexual como forma de violencia de género.

Y el siguiente objetivo específico:

- Exponer los indicios de identificación de posibles víctimas de trata de seres humanos con fines de explotación sexual.

Este trabajo está basado en la experiencia directa con mujeres próximas a redes de trata durante su estancia en el CETI la ciudad de Melilla de la investigadora, así como de la formación recibida en trata de personas con fines de explotación sexual impartida por la entidad Accem. Durante todo este proceso de experiencia vital y profesional hemos podido acceder a la observación directa no participante con estas personas, y a cierta documentación para la gestión de la atención de las mismas.

La trata de seres humanos constituye una grave violación de los Derechos Humanos Universales (Accem, 2019).

Según las Naciones Unidas, España se encuentra entre los principales países de tránsito y de destino de la trata, sobre todo de mujeres y niñas con fines de explotación sexual (Accem, 2019).

La definición de trata de seres humanos fue normativizada por primera vez en el denominado Protocolo de Palermo en el año 2000 por la Convención de las Naciones Unidas contra la Delincuencia Organizada Transnacional. Este documento aborda el tema de la trata desde la perspectiva de la criminalización del delito (Accem, 2019). 
Los elementos diferenciadores y característicos de esta definición son los siguientes:

- La acción de captar, transportar, trasladar, acoger o recibir personas.

- Recurriendo a la amenaza o al uso de la fuerza, a la coacción, al rapto, al fraude, al engaño, al abuso de poder o de una situación de vulnerabilidad o a la concesión o recepción de pagos o beneficios a una persona que tenga autoridad sobre la víctima.

- Con fines de explotación, lo que incluye la explotación de la prostitución ajena, la explotación sexual, los trabajos forzados, la esclavitud o prácticas análogas a la esclavitud y la extracción de órganos.

Con frecuencia, los términos trata de personas y tráfico ilícito de personas son confundidos. Por ello, hemos trabajado las diferencias entre ambos términos.

Las diferencias entre los términos trata y tráfico ilícito de personas son los siguientes:

- Consentimiento: En la trata de personas no hay consentimiento de traslado y si lo hay, éste es inválido al haberse obtenido mediante manipulación, engaños y coacción. No lo hay para la explotación. En el tráfico ilegal, sí existe consentimiento. Aún a pesar de las penosas condiciones que se pueden sufrir durante el transporte.

- Tipo de migración: En la trata puede ser regular o irregular. En el tráfico ilegal, sólo irregular.

- Transnacionalidad: En la trata puede ser transnacional o interna (trata doméstica). En el tráfico ilegal, sólo es transnacional.

- La explotación: En la trata sí es necesaria. Es la finalidad de la trata de seres humanos. Una vez cruzada la frontera, la víctima no es libre para terminar la relación con el tratante. En el tráfico ilegal, no es necesaria la explotación. El objetivo es la entrada en el país de destino. Una vez cruzada la frontera acaba la relación entre el traficante y el migrante.

- Fuente de beneficio: En la trata procede de la explotación. En el tráfico ilegal, procede del traslado.

- Bien jurídico protegido: En la trata, es el individuo. Es un delito mucho más grave. Atenta contra los derechos fundamentales y la dignidad de la persona. En el tráfico ilegal, son las normas de inmigración de los Estados. Es sobre todo un delito contra el Estado y el objetivo prioritario es garantizar el control de flujos migratorios.

- Participación de un grupo delictivo organizado: En la trata, no es necesario. Existe la trata nacional, e incluso local, en la que las personas captadoras 
pueden ser familiares, y no necesariamente un grupo organizado. En el tráfico ilegal, no es necesario.

Es en 2005 cuando la Unión Europea asume en su agenda política el tema de trata en el Convenio de Varsovia. Y el Estado Español realizará lo propio por primera vez en el 2007 con el Plan integral de lucha contra la trata de seres humanos con fines de explotación sexual. Análisis de situación y plan de actuación.

El Grupo de Expertos del Consejo de Europa (GRETA), encargado de realizar el seguimiento y evaluación de la aplicación del Convenio de Varsovia en los Estados Parte, insta a las autoridades españolas a la adopción de medidas integrales para garantizar la lucha contra la trata (Accem, 2019).

Teniendo muy presente esta recomendación, es muy importante cómo detectar a las víctimas de trata. Es importante que se les facilite el contacto de alguna organización o entidad con experiencia acreditada en asistencia para que les informe y/o acompañe. Los recursos de asistencia a su disposición pueden proporcionales: alojamiento seguro, alimentación e higiene personal, asistencia psicológica, asistencia médica, servicios de interpretación y asesoramiento jurídico. La estructura de esta atención es coordinada por un o una trabajadora social que tramita las gestiones pertinentes para poner en conocimiento del trabajador o trabajadora social de la entidad, la dispensación de atención psicológica, jurídica, educación social y trabajo social para las víctimas.

El área psicológica se encarga de recibir el ingreso, la evaluación correspondiente y ofrecer atención psicológica personalizada a cada víctima.

El área jurídica se encarga de explicar sus derechos a las víctimas, gestionar cualquier causa abierta, tramitar los documentos necesarios, y gestionar los aspectos legales en general.

El área de Educación Social se encarga de acompañar a las víctimas a cualquier asistencia necesaria, formación y verificar los cursos, etc. Se emiten informes para el área de psicología y el de Trabajo Social.

El área de Trabajo Social se encarga de toda la parte burocrática, gestionar los cursos, las formaciones, empleabilidad, etc. Debe trabajar conjuntamente con el coordinador para recibir información a la que quizá no tenga acceso.

Es responsabilidad del Cuerpo Nacional de Policía la identificación formal de las víctimas de trata de seres humanos.

La legislación nacional está compuesta por:

- Protocolo Marco de Protección de las víctimas de trata de seres humanos, de octubre de 2011 adoptado mediante acuerdo entre el Ministerio de Justicia, el Ministerio de Trabajo e Inmigración, el Ministerio de Sanidad, Política Social e 
Igualdad, el Consejo General del Poder Judicial y el Ministerio Fiscal que según su propio documento persigue como objetivo general "establecer pautas de actuación para la detección, identificación, asistencia y protección de las víctimas de trata de seres humanos, favorecer la coordinación de las instituciones implicadas en dichos procesos y definir los mecanismos de relación entre las administraciones con responsabilidades en la materia, así como, los procesos de comunicación y cooperación con organizaciones y entidades con experiencia acreditada en la asistencia a las víctimas de trata, en particular, aquellas que proporcionan una asistencia de carácter integral y participan en los programas de las administraciones públicas para la asistencia y protección de las mismas.

Es difícil acceder a este Protocolo debido a que sólo es posible acceder a él si se forma parte de un programa de intervención social subvencionado por el Estado Español, y de manera confidencial.

- Tipificación del delito de trata de seres humanos: Registrada en la Ley Orgánica 10/1995, de 23 de noviembre, del Código Penal, artículo 177 bis.

- La legislación correspondiente a la protección de las víctimas de trata en el Estado Español corresponde a:

- Ley Orgánica 4/2000, de 11 de enero, sobre derechos y libertades de los extranjeros en España y su integración social: Artículo 59 bis.

- Reglamento de la Ley Orgánica 4/2000, sobre derechos y libertades de los extranjeros en España y su integración social, aprobado mediante Real Decreto 557/2011, de 20 de abril. Ley 4/2015, de 17 de abril, del Estatuto de la Víctima del Delito que reconoce una serie de derechos procesales y extrapocesales para todas las víctimas de delitos, dando respuesta jurídica y social tanto a las víctimas como a sus familiares. Además de una atención específica hacia las víctimas más vulnerables, como son las víctimas de trata y las víctimas menores de edad.

- Real Decreto Ley $3 / 2013$, de 22 de febrero, por el que se modifica la Ley $1 / 1996$, de 10 de enero, de asistencia jurídica gratuita.

- Real Decreto 1192/2012, de 3 de agosto, por el que se regula la condición de asegurado y de beneficiario a efectos de la asistencia sanitaria en España, con cargo a fondos públicos, a través del Sistema Nacional de Salud, modificado por el Real Decreto 576/2013, de 26 de julio. 
- Ley 19/1994, de 23 de diciembre, de protección a testigos y peritos en causas criminales.

- Ley 35/1995, de 11 de diciembre, de Ayuda y Asistencia a Víctimas de Delitos Violentos y Contra la Libertad Sexual.

El análisis de los indicios para la identificación de posibles víctimas de trata de seres humanos con fines de explotación sexual es el resultado final de la experiencia directa de la docencia impartida en Español como segunda lengua, y su cultura, en el CETI de Melilla.

Durante el desarrollo de las clases también se impartieron talleres de inteligencia emocional y de resolución alternativa de conflicto tras la oportuna detección de estas necesidades. Toda la docencia impartida fue muy bien recibida por las alumnas, contando con una alta participación. Las alumnas procedían de Nigeria y tenían unas edades comprendidas entre los 20 y los 30 años. La metodología utilizada fue la observación no participante.

La utilización de una observación no participante permitió a la investigadora posicionarse frente a las personas inmigrantes residentes del CETI de Melilla. Decidimos este tipo de observación por varias razones: Una de ellas es para no interferir en su respuestas en el comportamiento. Por otra parte, las participantes son muy cautas a la hora de hablar, hemos de tener en cuenta que vienen de una ruta migratoria larga y dura, en la que han tenido que hacer y vivir cosas "no muy bien vistas" socialmente ni muy buenas para seguir adelante. Para ellas, tener confianza con una persona desconocida y de otra cultura es difícil. También tenemos que respetar sus tiempos, cada persona habla de su historia cuando está preparada para ello, y no cuando a los entrevistadores y entrevistadoras nos conviene, ya que, muchas de ellas han sido engañadas y abusadas por personas en las que confiaron. Incluso algunas pueden estar perseguidas.

La herramienta utilizada por la investigadora fue el diario de campo, que le sirvió para registrar los datos que iba recabando, así como, las impresiones y sistematizar tanto los hechos sucedidos como toda la información recogida hasta el momento. $Y$ en este punto, reflexionar sobre los siguientes talleres a programar, así como las clases de aprendizaje de Español y su cultura. Por tanto, el uso de nuestro diario ha sido eminentemente práctico.

El tipo de diario de campo utilizado se corresponde con el diario etnográfico, es decir, aunque el contenido de lo narrado es meramente descriptivo, tomamos en cuenta las consideraciones del contexto físico, social y cultural en el que se producían los hechos. Por tanto, los hechos registrados aparecen como parte de un conjunto más amplio de otros hechos que interactúan entre sí (Zabalza, 2005). Los hechos observados estaban comprendidos por connotaciones culturales, influenciados por la normativa correspondiente, -o por un vacío en ésta-, y por prácticas individuales. Por 
tanto, la reflexión realizada facilitaba la perspectiva diacrónica de la investigación llevada a cabo hasta el momento, me permitía reconocer los posibles obstáculos del próximo paso a realizar y la validez de toda la información recabada para la investigación de manera global.

Al término de cada día de investigación, la investigadora realizó el análisis de toda la información obtenida; identificaba los posibles obstáculos o problemas; seguimiento de temas de interés; particularidades; aproximación analíticas de las prácticas profesionales, -tanto propias como las observadas-; era más consciente de lo vivido y analizado; sistematizaba el o los próximos pasos a seguir a través de la toma de decisiones y los cambios pertinentes; e incluso registraba sentimientos y sensaciones vividas.

Dicho análisis se ha llevado a cabo a través de unos determinados parámetros. Siendo el resultado del mismo una compilación de todos los indicios detectados. Los parámetros fueron:

1.1-Indicios generales:

- En la fase de captación:

- Las víctimas proceden de un contexto de vulnerabilidad personal y/o familiar. Generalmente proceden de un entorno sociocultural y económico bajos. Pueden tener hijos/as en el país de origen.

- No organizó su viaje ni su documentación. También desconocía la ruta del viaje.

- Se le proporcionó información falsa sobre las condiciones de vida y/o trabajo en el país de destino.

- Ha podido ser captada mediante secuestro, a través de una relación sentimental o de amistad que le animó a emigrar. E incluso pudo haber sido captada por un familiar.

- Coste excesivo del viaje y deudas contraídas por el pago del mismo.

- Privación de libertad desde el momento de su captación hasta el pago de las deudas.

- Utilización de ciertos rituales religiosos, el vudú.

- Existencia de relación de dependencia respecto a una persona o grupo determinado.

- En la fase de transporte, traslado y recepción:

- No dispone de su documentación identificativa del país de procedencia o constan datos falsos. 
- Viajó acompañada de alguna persona o personas que le ayudaron a pasar controles fronterizos y le dieron instrucciones.

- Aislamiento social respecto de sus familiares y amigos/as. Su contacto se limita al entorno de la supuesta red y se encuentra bajo vigilancia.

- Dificultades para comunicarse en Español.

- No decide libremente su lugar de residencia y/o trabajo.

- En la fase de explotación:

- No tiene contrato o dispone de un contrato firmado en un idioma que desconoce y/o contiene condiciones abusivas de explotación.

- No dispone libremente de los ingresos derivados de su actividad.

- Restricción de libertad de movimientos.

- Es objeto de violencia psicológica, física y sexual, incluidas las amenazas.

- Incapacidad para mudarse a otro lugar.

- Vive y trabaja en el mismo lugar.

- Jornadas de trabajo excesivas.

- Amenazas y violencia tanta a las víctimas como a sus familiares.

- Amenaza de deshonrar a las víctimas revelando las circunstancias a su familia.

- Amenaza a las víctimas en caso de denuncia, muchas de ellas provienen de países donde se teme a las autoridades, y hay desprotección por parte de la ley.

- Amenazas por vinculación de delitos.

- Posesión de un móvil (uno o varios) o tarjeta SIM recién llegadas a España.

- Cambio de las expectativas sobre la vida en el destino.

- No contar con una red social de apoyo o conocidos en España, o en otros países europeos.

- Uso de móviles: Número de llamadas recibidas, frecuencia de llamadas.

- Discurso: Reticencia a hablar, contradicciones, incongruencias, ansiedad.

- Urgencia por abandonar el recurso de acogida, en el caso que cuente con éste. 
- Imposibilidad de mudarse a otro lugar.

- No suelen saber que son víctimas de trata de personas, no saben qué es.

- Suelen llegar en grupo, con la existencia de una persona que tiene el rol de "líder", quizá más mayor, que habla por todas, sabe todo y es persona de referencia para el grupo.

- Posible rol de madame.

1.2- Indicios psicológicos.

- Emociones llegadas a límite: Miedo, culpabilidad, ira, traición, desconfianza, desamparo, crisis nerviosa, inhabilidad de permanecer en un solo lugar, lagunas en la memoria.

- Depresión.

- Ansiedad.

- Nerviosismo.

- Trastornos psíquicos.

- Alteración del sueño.

- Ataques de pánico.

- Llora con facilidad.

- Pérdida de interés en las cosas.

- Adicciones a diversas sustancias estimulantes o depresoras.

1.3-Indicios físicos.

- Moratones.

- Cicatrices.

- Indicios de violación o abusos sexuales.

- Indicios de aborto.

1.4- Indicios conductuales.

- "Posesión de móvil recién llegadas a España.

- Reticencia o desgana para hablar.

- Manifestaciones incoherentes o que indican adoctrinamiento.

- Comportamiento alterado, nerviosismo y otros síntomas que inducen a pensar que se encuentran bajo una situación de abuso, malos tratos y/o amenazas. 
- Muestra miedo o ansiedad en presencia de personas con quienes viajó a España. En ocasiones intervienen como intérpretes.

- Existe relación de dependencia respecto a una persona o grupo determinado.

- Recibe visitas de supuestos conocidos/familiares, constantes llamadas al móvil y/o paquetes a su nombre en el dispositivo de acogida.

- Apariencia desaliñada y descuidada.

- Conducta temerosa e impresionable (sudor, temblor, dudas para responder preguntas directas, evita contacto visual por cuestiones ajenas a las culturales).

- Expresa miedo a represalias o preocupación por la situación de sus familiares.

- Las víctimas están forzadas a hacer cosas en contra de su voluntad.

- Salidas y entradas en el dispositivo de acogida en horarios que no se corresponden con la actividad cotidiana" (Ministerio de Empleo y Seguridad Social, anexo 1).

La determinación de estos indicios son claves para conocer cómo y por qué una mujer puede caer en manos de redes de trata de personas. De igual manera, facilita conocer el modus operandi de las redes, y cómo estructurar la entrevista adecuada para detectar a una posible víctima.

A este respecto, es muy importante tener en cuenta en la estructuración de las entrevistas lo siguiente:

- Presentación de la persona entrevistadora y cargo que desempeña.

- Explicar el papel de la persona intérprete.

- Explicar la finalidad de la entrevista.

- Explicar los pasos a dar y la necesidad de los mismos.

- Empoderar a la persona entrevistada: Informarla sobre su derecho a no contestar, a interrumpir o poner fin a la entrevista cuando quiera.

- Intentar conocer qué espera la persona de la entrevista.

- Dar espacios para que la persona entrevistada pueda hacer preguntas.

- Estar preparados/as para responder a posibles muestras de angustia y aflicción.

- Aprovechar la necesidad de registro de los datos para formular determinadas preguntas y no volver a repetirlas (nombre, edad, pasaporte ...).

- Terminación de la entrevista: 
- En el mejor de los casos: Acordar seguimiento, próximo encuentro, acompañamiento y derivación a otros recursos. Asegurarse de que se ha entendido todo lo que queremos transmitir.

- En el peor de los casos: Dotar a la persona entrevistada de la máxima información de forma clara y sencilla para que pueda ponerse en contacto en cualquier momento, a corto o largo plazo.

\section{Conclusiones}

Las conclusiones obtenidas del objetivo específico:

- Exponer los indicios de identificación de posibles víctimas de trata de seres humanos con fines de explotación sexual.

Los indicios para la identificación de estas posibles víctimas pueden dividirse en:

- Indicios generales.

- Indicios psicológicos.

- Indicios físicos.

- Indicios conductuales.

Los indicios generales se dividen a su vez en:

- Indicios en la fase de captación: Relacionados con las características del contexto procedente de las personas captadas. Así como, con las personas que organizaron el viaje y los medios utilizados para conseguir que la víctima viaje, con o sin consentimiento de ésta.

- Indicios en la fase de transporte, traslado y recepción: Relacionados con la propia organización del viaje y la documentación necesaria.

- Indicios en la fase de explotación: Relacionados con las condiciones propias de la actividad que tendrá que desarrollar la víctima bajo sometimiento y algún tipo de violencia.

Los indicios psicológicos pueden detectarse porque se traducen en la práctica en enfermedades, trastornos y alteraciones psicológicas derivadas de las emociones llevadas al límite. Emociones como miedo, culpabilidad, ira, traición, desconfianza, crisis nerviosa o lagunas en la memoria.

Estos indicios pueden ser depresión, ansiedad, nerviosismo, alteraciones del sueño, pánico, facilidad para llorar, pérdida de interés por las cosas o adicciones.

Los indicios físicos pueden resumirse a toda señal corporal que puedan detectarse derivadas de daños realizados, abusos o abortos.

Los indicios conductuales son los relacionados con el comportamiento. Un comportamiento propio de las personas a las que se les ha podido detectar el resto de 
indicios. Todos los indicios analizados se expresan a través de un comportamiento dado. Este comportamiento puede resumirse en reticencia para hablar, manifestaciones incoherentes, comportamiento alterado, apariencia descuidada, conducta temerosa e imprevisible, expresiones de miedo o preocupación, o salidas y entradas en el dispositivo de acogida.

Todos estos indicios son muy importantes tenerlos en cuenta para programar la estructura de las entrevistas.

La estructura de las entrevistas debe disponer de:

- Presentación: Tanto de la persona entrevistadora como la del intérprete. Así como, explicar el fin de la misma y los pasos a dar.

- Desarrollo de la entrevista: Informar sobre los derechos de la persona entrevistada, conocer qué espera esta persona de la entrevista, dar espacios para que ella pueda realizar sus preguntas, dar respuesta ante posibles muestras de angustia, y realizar registro de datos relevantes.

- Término de la entrevista: Puede acordarse la fecha del próximo encuentro, determinar el acompañamiento apropiado y/o la derivación a otros recursos. 0 bien, la entrevista puede culminarse proporcionando a la persona entrevistada de la máxima información que pueda necesitar para que pueda volver a ponerse en contacto en cualquier momento.

\section{Bibliografía}

Accem. (2019). La otra cara de la trata. Informe diagnóstico sobre otras formas de trata que afectan a las mujeres. Madrid España: Accem.

BOE. (1995). Ley 35/1995, de 11 de diciembre de ayudas y asistencias a las víctimas de delitos violentos y contra la libertad sexual. Núm. 296 - 35576. Recuperado de https://www.boe.es/buscar/doc.php?id=BOE-A-1995-26714

BOE. (1994). Ley Orgánica 19/1994, de 23 de diciembre, de protección a testigos y peritos en causas criminales. Núm. 307 - 28510. Recuperado de http://www.boe.es/boe/dias/1994/12/24/pdfs/A38669-38671.pdf

BOE. (1995). Ley Orgánica 10/1995, de 23 de noviembre, del Código Penal. Núm. $281-$ 25444. Recuperado de http://www.boe.es/boe/dias/1995/11/24/pdfs/A3398734058.pdf

BOE. (2000). Ley Orgánica 4/2000, de 11 de enero, sobre derechos y libertades de los extranjeros en España y su integración social. Núm. 10 - 544. Recuperado de http://www.boe.es/boe/dias/2000/01/12/pdfs/A01139-01150.pdf

BOE. (2011). Real Decreto 557/2011, de 20 de abril, por el que se aprueba el Reglamento de la Ley Orgánica 4/2000, sobre derechos y libertades de los extranjeros en España y su integración social, tras su reforma pro Ley Orgánica 2/2009. Núm. 103 - 7703. Recuperado de https://www.boe.es/buscar/pdf/2011/BOE-A-2011-7703consolidado.pdf 
BOE. (2012). Real Decreto 1192/2012, de agosto, modificado por el Real Decreto 576/2013, de 26 de julio. Núm. 186. Recuperado de http://noticias.juridicas.com/base datos/Admin/rd1192-2012.html

BOE. (2013). Real Decreto Ley 3/2013, de 22 de febrero, por el que se modifica la Ley 1/1996, de 10 de enero. Núm. 47 - 2029. Recuperado de https://boe.es/boe/dias/2013/02/23/pdfs/BOE-A-2013-2029.pdf

BOE. (2015). Ley 4/2015, de 27 de abril, del Estatuto de la víctima del delito. Núm. 101 - 36569. Recuperado de https://www.boe.es/boe/dias/2015/04/28/pdfs/BOE-A2015-4606.pdf

Consejo de Europa. (2005). Convenio del Consejo de Europa sobre la lucha contra la trata de seres humanos. Recuperado de http://www.accem.es/ficheros/documentos/pdf_trata/Convenio_Consejo_de_ Europa.pdf

Defensor del Pueblo. (2015). Informe anual 2015 y debates en las Cortes Generales. Recuperado de https://www.defensordelpueblo.es/wpcontent/uploads/2016/02/Informe2015.pdf

Ministerio de Empleo y Seguridad Social. (2011). Protocolo para la detección y actuación ante posibles casos de trata de seres humanos con fines de explotación sexual.

Ministerio de Igualdad. (2010). Plan integral contra la trata de seres humanos (20092011). https://tbinternet.ohchr.org/Treaties/CEDAW/Shared\%2oDocuments/ESP/INT _CEDAW_NGO_ESP_61_18795_S.pdf

Ministerio del Interior. (2007). Plan integral de lucha contra la trata de seres humanos con fines de explotación sexual. Análisis de situación y plan de actuación. Recuperado http://www.intermigra.info/extranjeria/archivos/impresos/PlantrataMIR.pdf

Ministerio de Sanidad, Servicios Sociales e Igualdad (2015). Plan Integral de lucha contra la trata de mujeres y niñas con fines de explotación sexual (2015-2018). Recuperado de: http://www.violenciagenero.msssi.gob.es/planActuacion/planContraExplotaci onSexual/docs/Plan_Integral trata_18 Septiembre2015 2018.pdf

Naciones Unidas. (2000). Protocolo para prevenir, reprimir y sancionar la trata de personas, especialmente mujeres y niños. Recuperado de https://www.ohchr.org/documents/professionalinterest/protocoltraffickinginp ersons_sp.pdf

Zabalza Beraza, M. A. (2004). Diarios de clase: Un instrumento de investigación y desarrollo profesional. Madrid, España: Narcea. 


\section{Cómo referenciar este artículo/How to reference this article $(*):$}

Lara Palacios, M.A., Monreal Gimeno, M.C., y Sánchez Fernández, S. (2021). Trata de mujeres con fines de explotación sexual y violencia de género. Indicios para la detección de posibles víctimas. iQUAL. Revista de Género e Igualdad, 4, 109-123, doi: 10.6018/iqual. 445011

Lara Palacios, M.A., Monreal Gimeno, M.C., y Sánchez Fernández, S. (2021). [Trafficking in women for sexual exploitation purposes and gender violence. Clues for the detection of possible victims]. iQUAL. Revista de Género e Igualdad, 4, 109-123, doi: 10.6018/iqual. 445011

(*) La autoría colectiva responde a una contribución conjunta en todos los apartados. 